\title{
Effects of independent outcome-values of past events upon subsequent choices ${ }^{1}$
}

\author{
FRANCIS W. IRWIN AND MARSHA JOAN METZGER \\ UNIVERSITY OF PENNSYLVANIA
}

Choices made by human Ss in two experiments showed that their beliefs as to which of two alternative outcomes of a probabilistic event had occurred in the past were affected by the preference values that these outcomes possessed prior to the occurrence of the event.

It has been shown repeatedly (Irwin \& Snodgrass, 1966; Irwin \& Metzger, 1966; and others) that predictions of events are positively related to values that depend only upon the occurrence of the events themselves and not upon the predictions. These values are called independent outcome-values (IO), in contrast to dependent outcome-values (DO), which depend upon both the events and the predictions.

If the effects of 10 are interpreted as influences of the values of future events upon the expectancies that they will occur, it can be asked whether IO has similar effects upon beliefs concerning past events. Consider, for example, an experiment in which $\mathrm{S}$ draws one card from each of two identical shuffled packs, the constitution of which he knows, under conditions in which drawing a card marked with an $X$ is preferable to drawing a blank card in the case of one pack (Pack $M+$ ), whereas the reverse is true of the other pack (Pack M-). Then, according to the above interpretation, $S$ will have a stronger expectancy of drawing a marked card from Pack $M+$ than . from Pack $M-$. Suppose that $S$ now makes the draws, keeping the cards face down. Then, unless the process of drawing affects S's assessment of the situation, he should believe that the card he drew from Pack M+ is more likely to be a marked card than is the one he drew from Pack $M$ - and, if he is required to choose between them and is paid off for choosing a marked card rather than a blank, he should choose the card from $M+$ over the card from M-. The following two experiments tested this conclusion.

Subjects

The Ss were undergraduates at the University of Pennsylvania. There were 15 males and 15 females in Experiment 1 and 16 males and 16 females in Experiment 2.

\section{Procedure}

Experiment 1. The Ss were seen individually and were told they were to play a "decision-making game." Packs of cards of playing-card size were used. Each pack contained 10 cards, some blank and some with a large boldface $X$ printed on the face ("marked cards"). The packs contained 1, 3, 5, 7, or 9 marked cards, and each of the 25 possible pairs of such packs was used once with each S. On each trial, $S$ was informed of the constitution of the two packs used on that trial orally and by means of a typed slip. In addition, he was informed in the same ways that if he drew a marked card from a designated one of the two packs (Pack $M+$ ), he would win 10 cents, whereas if he drew a marked card from the other pack (Pack M-), he would lose 10 cents. Blank cards always had a value of zero. $S$ drew a card face down from each pack. He then chose one of the two drawn cards with the understanding that if the chosen card was marked, he would win 10 cents in addition to the payoffs for the draws, and if it was blank, its value was zero. Payoffs and feedback were given only at the end of the session. Packs $\mathbf{M}+$ and $\mathbf{M}$ - were alternately on the left and the right from trial to trial, and a different pseudorandom order of packs and pairs was used for each $\mathrm{S}$.

Experiment 2. The procedure was like that of Experiment 1 except for the following conditions. (1) The numbers of marked cards in the pairs of packs $\left(M_{+}, M^{-}\right)$were: 3,$3 ; 3,4 ; 4,3 ; 4,4 ; 4,5 ; 5,4$; 5,$5 ; 5,6 ; 6,5 ; 6,6 ; 6,7 ; 7,6 ; 7,7$. Each pair was used twice for each $S$, once with $M_{+}$on the left and once with $\mathrm{M}+$ on the right. In addition, $(3,4)$ was used once with both packs being $M+$ and once witheboth being $\mathrm{M}-$; the same was true of $(6,7)$. (2) The payoffs for the cholce between the two drawn cards were winning 5 cents if the chosen card was marked and losing 5 cents if it was blank.

\section{Results and Discussion}

The results of Experiments 1 and 2, combined over Ss, are given in Figs. 1 and 2. The diagonals show the percents of choices of the card that had been drawn from Pack $\mathrm{M}+$ when the probabilities of having drawn a marked card, $P(M)$, from Packs M+ and M- were equal. It can be seen that the Ss strongly tended to choose the card that had been drawn from Pack M+, all five percents being greater than 50 in each experiment. The cells above and below the diagonal in each figure contain the percents of choices of the card that had been drawn from the pack with the larger $P(M)$, in the case of pairs in which $P(M)$ differed. (Note that these percents are defined differently from those in the diagonals.) All of these values are greater than 50 percent and, as expected, they tend to increase with the difference in number of marked cards between the packs. In addition, however, it can be seen in each figure that the card from the pack with the larger $P(M)$ 


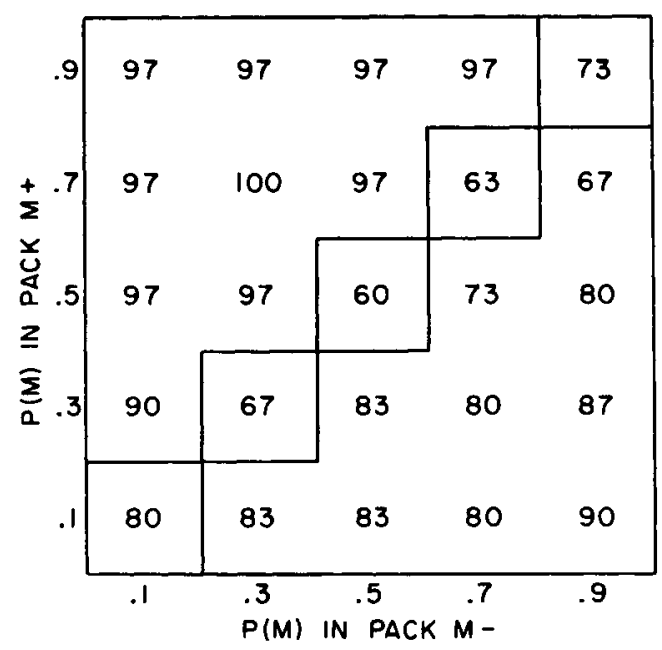

Fig. 1. Experiment 1. Diagonal: percent choices of card from Pack $M+$ when $P(M)$ was the same in the two packs. Above and below diagonal: percent choices of card from pack with greater $P(M)$. Each entry is based upon one choice by each of $30 \mathrm{Ss}$.

was chosen more often when that pack was $M+$ than when it was M-, given that the probabilities were the same in the two pairs of packs. For example, consider in Fig. 1 the pairs of packs in which $P(M)$ was .7 for one pack and .3 for the other, and note that the card from .7 was chosen in 100 percent of the cases when $M+$ was .7 and $M-$ was .3 , and in only 80 percent when $M-$ was .7 and $M+$ was .3. A difference in this direction is present in all 10 comparisons of this sort in Fig. 1 and all four in Fig. 2. Finally, recall that in Experiment 2 there were pairs of packs in which the members were sometimes both $\mathrm{M}+$ and sometimes both $\mathrm{M}-$. When both members were $M+$, 88 percent of the choices were of the card from the pack with the greater $P(M)$, but when both members were $\mathrm{M}-$, the percent was only 67 . This means that the Ss tended to choose against the probability more often when the less probable outcome was originally favored by its IO than when the reverse was the case.

Considering the individual Ss, 44 of the 62 in the two experiments taken together chose the card from Pack M+ more often than that from M-, 15 did the reverse, and three chose equally often from the two packs. Tested by the standard error of a frequency, these results differ significantly $(p<.01)$ from symmetrical distribution. In Experiment 2, each $S$ could be given two scores, one for the number of choices of the card from Pack $M+$ when this was on the right and one for the cases in which $M+$ was on the left. An analysis of variance of these scores showed that

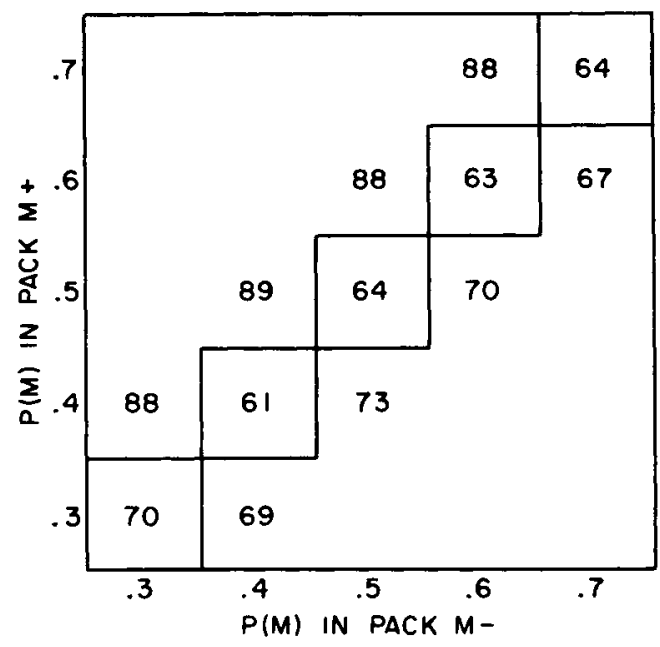

Fig. 2. Experiment 2. Diagonal: percent choices of card from Pack $M+$ when $P(M)$ was the same in the two packs. Above and below diagonal: percent choices of card from pack with greater $\mathbf{P}(\mathbf{M})$. Each entry is based upon two choices by each of 32 Ss.

neither sex nor the left-right variable had effects approaching significance, but that differences between Ss were highly significant $(p<.1)$. The product moment correlation between the Ss' scores was .73 $(\mathrm{N}=32)$.

It is clear that the differential values of drawing marked cards from the two packs of a pair affected the subsequent choices between the two drawn cards. The effect was consistent with the hypothesis that the Ss were more likely to believe that a previously drawn card was of a particular kind (marked or blank) if having drawn that kind was desirable than if it was undesirable. If, as the hypothesis supposes, such differences in strength of belief resulted from corresponding differences in the expectancies prior to the draws, the influence of IO upon these expectancies cannot be ascribed to rewarding or punishing effects of making overt bets and predictions. No such predictions were made and, even if the choices are taken to be bets, they occurred after the presumed effect of IO had taken place.

\section{References}

IRWIN, F. W., \& SNODGRASS, J. G. Effects of independent and dependent outcome values upon bets. J. exp. Psychol., 1966, 71, 282-285.

IRWIN, F. W., \& METZGER, M. J. Effects of probabilistic independent outcomes upon predictions. Psychon. Sci. 1966, 5, 79-80.

Note

1. This investigation was supported by Research Grant MH-06580 from the National Institute of Mental Health to the University of Pennsylvania. We thank Allen H. Harris, Jr. for assistance in obtaining data and Thomas $\mathrm{S}$. Wallsten for helpful comments. 